sierodiagnosi può fornire un valido contributo. In caso di infezione sospetta la conoscenza della prevalenza degli anticorpi nella popolazione normale (donatori di sangue) è molto importante per l'interpretazione dei risultati degli esami sierologici.

\title{
PREVALENZA DI ANTICORPI ANTI- YERSINIA IN DONATORI DI SANGUE.
}

\author{
Franzin L.', Bonfrate N.', Caboti D.', Gallo P.' \\ ' Laboratorio "Ricerca Speciale Microbiologica", \\ Ospedale Amedeo di Savoia, Corso Svizzera 164, 10149 Torino. ${ }^{2}$ \\ Centro Immunoematologia e Trasfusionale, \\ Ospedale Maria Vittoria, Via Cibrario 72, 10144 Torino.
}

Introduzione. Yersinia enterocolitica può essere responsabile di gastroenterite, ileite terminale, linfoadenite mesenterica e pseudoappendicite.

Il batterio è causa sempre più frequente di shock settico posttrasfusionale. Scarsi sono gli studi sulla prevalenza di anticorpi nei donatori di sangue. Scopo del lavoro è valutare il titolo anticorpale contro i ceppi patogeni di Yersinia più frequentemente isolati in Europa in un gruppo di donatori di sangue.

Metodi. Sono stati esaminati 407 sieri di donatori dell'area torinese. Gli anticorpi anti- Y. enterocolitica O:3, O:5, O:9 e $Y$. pseudotuberculosis I sono stati determinati con il metodo di microagglutinazione mediante l'utilizzo di antigeni standardizzati preparati nel nostro Laboratorio con ceppi di riferimento, gentilmente forniti dal Prof. H. H. Mollaret, Institut Pasteur, Parigi.

Risultati. L'età dei donatori era compresa tra 18 e 74 anni, di cui $81.8 \%$ maschi. Sono stati riscontrati titoli anticorpali da 1/10 a 1/40 per $Y$. enterocolitica O:3, O:5, O:9 e Y. pseudotuberculosis I rispettivamente in $12.8,32.2,8.3$ e $34.4 \%$ dei sieri. Titoli anticorpali elevati sono stati trovati in percentuali inferiori (1/80 in solo 5 casi, 1/160 e 1/320 in un caso). Bassi titoli anticorpali (1/10-1/20) sono stati osservati in 11\% dei campioni per $Y$. enterocolitica O:3, in $29.5 \%$ per $Y$. enterocolitica $\mathrm{O}: 5$, in $6.4 \%$ per $Y$. enterocolitica $\mathrm{O}: 9$ e in $33.2 \%$ per Y. pseudotuberculosis .

\section{Conclusioni.}

Nella popolazione esaminata gli anticorpi anti-Yersinia sono presenti in maniera significativa, seppur a titoli bassi. La diagnosi di Yersiniosi è raggiunta in maniera ottimale con l'isolamento del microrganismo. In mancanza di questo esame la 